Original

Article

\title{
Impact of Medical Treatment on Long-Term Results after Surgical Ablation of Atrial Fibrillation in Cardiac Surgical Patients
}

\author{
Richard Feyrer, MD, MBA, Fatos Ballazhi, MD, Timo Seitz, MD, \\ Michael Weyand, MD, and Frank Harig, MD
}

\begin{abstract}
Background: The aim of this study was to evaluate the long-term outcome (4 years) of high-intensity-focused-ultrasound (HIFU) cardiac ablation, the significance of postablation antiarrhythmic drugs (AADs) and predictors of successful sinus rhythm (SR) restoration. Methods: 103 patients were prospectively enrolled in a single-center study. The preoperative atrial fibrillation (AF) type was paroxysmal in $36 \%$, permanent in $53 \%$, persistent in $5 \%$ and flutter in $6 \%$ patients. The left atrial diameter was $<\mathbf{5 0} \mathbf{~ m m}$ in 78 patients and $>\mathbf{5 0} \mathbf{~ m m}$ in 25. Long-term results, up to 4 years, and postablation antiarrhythmics were evaluated. Follow-up studies including 12-lead electrocardiogram (ECG) and 24-h Holter ECG obtained at 3 and 6 months in our institute, and 12, 24, and 48 months during outpatient visits.

Results: No device- or procedure-related complications or deaths were observed. A pacemaker was implanted in 5\% patients. Freedom from AF and flutter at 6-/12-/and 48month visit were $66 \% / 63 \% / 68 \%$ for the entire population, $84 \% / 77 \% / 90 \%$ in patients with paroxysmal $\mathrm{AF}, 50 \% / 46 \% / 40 \%$ in patients with permanent $\mathrm{AF}$ and at any time in all 6 patients with flutter. No significant changes were noted at 2 years.

Postablation results were not statistically different in patients on or not on antiarrhythmic therapy. At discharge, $53 \%$ of patients on antiarrhythmics and 55\%, not on AADs were free of AF. At the 6-month visit, $66 \%$ of patients on AADs versus $69 \%$ of patients, not on AADs presented with SR, an improved outcome by 13 percentage points with AADs versus 14 percentage points without AADs $(p=0.65)$. Patients taking a $B$-blocker showed better results in restoration/maintenance of $S R$ after 6 months. No significant difference was noted regarding the use of UltraWand.

Sixty-seven percent with a left atrium (LA) diameter $<\mathbf{5 0} \mathbf{~ m m}$ were successfully converted into SR, vs. $48 \%$ with an LA diameter $>50 \mathrm{~mm}$. Best results were achieved in patients with paroxysmal $\mathrm{AF}$ and $\mathrm{LA}<\mathbf{5 0} \mathbf{~ m m}$ with $81 \%$ freedom of AF.

Conclusion: AF treatment with HIFU ablation during concomitant cardiac surgery is a safe and effective procedure for restoring SR, especially in patients with AF and especially in patients with a smaller left atrial diameter. No significant difference was noted between the antiarrhythmic and non drug groups regarding restoration and maintaining SR; however, better results were achieved by those taking a $B$-blocker.
\end{abstract}

Keywords: atrial fibrillation, surgical ablation, HIFU, antiarrhythmic drugs

Center of Cardiac Surgery, University Hospital of Erlangen, Erlangen, Germany

Received: December 20, 2012; Accepted: January 16, 2013

Corresponding author: Richard Feyrer, MD, MBA. Center of
Cardiac Surgery, University Hospital of Erlangen, Krankenhausstr 12, 91054 Erlangen, Germany Email: richard.feyrer@uk-erlangen.de (C)2014 The Editorial Committee of Annals of Thoracic and Cardiovascular Surgery. All rights reserved. 


\section{Introduction}

Atrial fibrillation (AF) is a widespread cardiac rhythm disorder. The prevalence of AF rises with advancing age, from $2 \%$ in persons over the age of 40 , to $6 \%$ in persons over the age of 70 and about $15 \%$, in those over the age of $90 .{ }^{1)}$ The number of people with this cardiac arrhythmia has increased considerably during the past 30 years. In Germany, almost 1 million people, in Europe over 6 million and in the United States, some 2.3 million people are affected. ${ }^{2,3)}$

$\mathrm{AF}$ is associated with increased rates of death, stroke and other thrombo-embolic events, heart failure and hospitalizations, degraded quality of life, reduced exercise capacity and left ventricular (LV) dysfunction. ${ }^{2)}$ Stroke remains the most feared complication. In persons with coronary heart disease or cardiac failure, AF doubles the stroke risk in men and trebles the risk in women. ${ }^{4)}$ AF currently represents a major economic burden for society in light of the aging population. ${ }^{5-7)}$

The Maze procedure provides excellent results for surgical treatment of AF. ${ }^{8)}$ This surgical approach was designed to block multiple macroreentrant circuits that were the putative cause of AF. Due to its relative complexity and invasiveness, the Cox-maze procedure has not been widely adopted by the surgical community.

Focal mechanisms potentially contribute to the initiation and perpetuation of AF. The pulmonary veins were identified as major sources of atrial triggers (ectopic beats). ${ }^{9)}$

A number of ablation methods using different sources of energy, including radiofrequency, microwave, laser and cryotherapy, have been developed to create intraatrial lesion lines around the pulmonary orifices, either endocardial or epicardial. The reasons for failure of the method are still unknown exactly. ${ }^{10-14)}$

The Epicor Medical approach, using therapeutic ultrasound in form of high intensity focused ultrasound (HIFU) applied on the epicardial side of the left atrium (LA) is a safe and effective energy source that allows surgeons to replicate the mini-maze lesion set precisely on a beating heart. ${ }^{15}$ )

In our single experience study were evaluated the clinical long-term outcomes (ranging to 4 years) after HIFU ablation during concomitant cardiac surgery, the significance of postablation antiarrhythmic drug (AAD) therapy, the safety and efficacy of UltraWand and the assessment of predictors for a successfully restoration of sinus rhythm (SR).

\section{Materials and Methods}

In concomitant cardiac surgery, 103 patients underwent an LA ablation procedure using HIFU (Epicor) technology at University Hospital, Erlangen, Germany, from March 2007 to December 2011. The type and distribution of preoperative AF were paroxysmal in $37(36 \%)$, permanent in $55(53 \%)$, persistent in $5(5 \%)$ and flutter in $6(6 \%)$ patients. LA diameter measured at preoperative echocardiography was $<50 \mathrm{~mm}$ in 78 (76\%) and $>50 \mathrm{~mm}$ in 25 (24\%) patients. The detailed primary concomitant procedures are summarized in Table 1.

\section{System description}

The Epicor Medical Cardiac Ablation System is designed to deliver HIFU via an entirely epicardial approach and consists of an array of transducers (UltraChinc) positioned after proper sizing around the LA wall of the pulmonary vein orifices and of a Handheld device (UltraWand) to create the mitral line component of the Cox-maze procedure extending from the left lower vein orifice to the mitral valve annulus. Furthermore, technical specifications are described at the developer Site, St. Jude Medical [www.sjm.com].

\section{Ablation procedure}

AF ablation was performed after the sternotomy and heparinization by inserting the Epicor device circumferentially around the pulmonary veins on the beating heart prior to the commencement of cardiopulmonary bypass for concomitant cardiac procedures, during an approximately 10-minute algorithm as described elsewhere. A mitral line lesion was also performed in 36 cases after lifting the apex of the heart and exposing the posterior pulmonary veins. Once exposure was obtained, the hand held device (UltraWand), using the same ultrasonic technology, was activated to connect the previously created circumferential line to the mitral annulus in the line of the left inferior pulmonary vein.

\section{Post-ablation treatment}

After cardiopulmonary bypass, all patients were treated with intravenous amiodarone for at least 24 hours. Amiodarone administration was started with a bolus of $300 \mathrm{mg}$, followed by an infusion of $24-72 \mathrm{mg} / \mathrm{h}$ for up to 3 days. The anticoagulation therapy was started with intravenous heparin 6 hours after surgery. All patients received continuous bedside electrocardiogram (ECG) monitoring for about 7 days. In patients with paroxysmal 
Table 1 List of concomitant cardiac procedures in 103 patients

\begin{tabular}{lrr}
\hline Procedures & $\mathrm{n}$ & $\%$ \\
\hline MV-surgery (isolated) & 18 & 17.5 \\
MV-surgery associated with CABG & 3 & 3 \\
MV-surgery associated with AVS & 2 & 2 \\
AV-surgery & 34 & 33 \\
AV-surgery associated with CABG & 8 & 8 \\
Tricuspid valve surgery & 3 & 3 \\
Other & 2 & 2 \\
Total & 103 & \\
\hline
\end{tabular}

MV: mitral valve; CABG: coronary artery bypass graft; AVS: aortic valve surgery; $\mathrm{AV}$ : aortic valve

and permanent $\mathrm{AF}$, anticoagulation (warfarin or coumadin) and antiarrhythmics were switched to oral medication and maintained for at least 3 months. Amiodarone therapy was given to patients with permanent $\mathrm{AF}$ and poor prognosis to restore a SR. Patients with high heart rate frequency or often occurred paroxysmal AF received single amiodarone therapy or associated with B-blocker. Patients with bradycardia or temporarily bradyarrhythmia absoluta were treated acute with Atropine, Orciprenaline, Theophylline or withdrawal of Antiarrhythmics. After 3 months in most patients were continued single antiarrhythmic therapy with a $\beta$-blocker. Amiodarone was recommended for maximally 6 months.

\section{Follow-up}

Follow-ups including physical examination, ECG or 24h-Holter ECG and echocardiography were obtained at 3- and 6-month in our institute. After 6 months and for as long 4 years, patient status was determined by screening records of outpatient visits and correspondence with referring physicians. Long term results were evaluated in 30 patients from the year 2007 and 2008. Follow-up of these groups was $93 \%$ at 3 months, $90 \%$ at 6 months and $80 \%$ at 4 years.

\section{Statistical analysis}

All data analyses were performed with the SPSS system for statistics (SPSS 18/19 for Windows, SPSS, Inc., Chicago, Illinois, USA). Clinical profiles were compared using the $\mathrm{Chi}^{2}$ or the Fisher exact test. All tests of significance were 2-sided. A probability value of $\leq 0.05$ was considered significant. For statistical analysis of long-term outcomes ranging to 4 year, patients from the year 2007 and 2008 were recruited.

\section{Results}

All 103 patients had successful feasibility of HIFU system on the beating heart prior to cardiopulmonary bypass. No device-related or procedure-related complications occurred, particularly no bleeding, injury of the oesophagus, pulmonary artery or veins. There were 6 early deaths by multi organ failure caused by cardiogenic shock in three cases, general sepsis after candida albicans pneumonia in one case and two patients died by mediastinitis. Late mortality consisted of 4 patients who died from noncardiac causes in 3 cases and in another case from mediastinitis. A permanent pacemaker was required in 5 patients, 4 for complete heart block and 1 for sick-sinus rhythm. In 3 patients were performed mitral valve surgery and in 2 patients aortic valve surgery.

Freedom from AF and flutter at discharge was $62 \%$ for the entire population, $49 \%$ in patients with permanent, $73 \%$ in patients with paroxysmal, $80 \%$ in patients with persistent $\mathrm{AF}$ and in all 6 patients with flutter. (Fig. 1)

At the 6-/12-/48-month visit 84\%/77\%/90\% of patients with paroxysmal AF, 50\%/46\%/40\% of patients with permanent $\mathrm{AF}$ and at any time in all 6 patients with flutter were presented with SR (Fig. 2). No significant changes were noted at 2 years. Long-term outcome of HIFU ablation ranging to 4 years were analysed in patients from 2007 and $2008(\mathrm{n}=30)$. Follow-up was $80 \%$.

For the entire population, $66 \%$ of patients were presented with SR at 6 month visit, $68 \%$ at 4 year visit. No significant changes were noted at 1 and 2 years.

No significant difference was noted regarding the use of UltraWand. $61 \%$ of patients using U-Wand were free of $\mathrm{AF}$, versus $63 \%$ without $\mathrm{U}$-Wand usage $\left[\mathrm{chi}^{2}=0.66\right.$ $(\mathrm{df}=1) ; \mathrm{p}=0.79]$.

We also compared the impact of postoperative (postablation) AADs versus patients who were discharged without antiarrhythmics. 55\% of no drug group were presented with SR at discharge versus $53 \%$ of antiarrhythmic group. In both patient groups were noted improved outcome in restoration/maintenance of $\mathrm{SR}$ at 6 month visit. No significance difference was noted between no drug group and AADs-group [p = $0.80]$.

Separately were evaluated the significance of antiarrhythmics, particularly ß-blocker, versus amiodarone, versus non drug therapy. In patients who were treated with B-blocker were seen better results in restoring/maintenance of SR, however, it was not significant. So as Fig. 3 shows, $51 \%$ of patients with postablation ß-blocker 


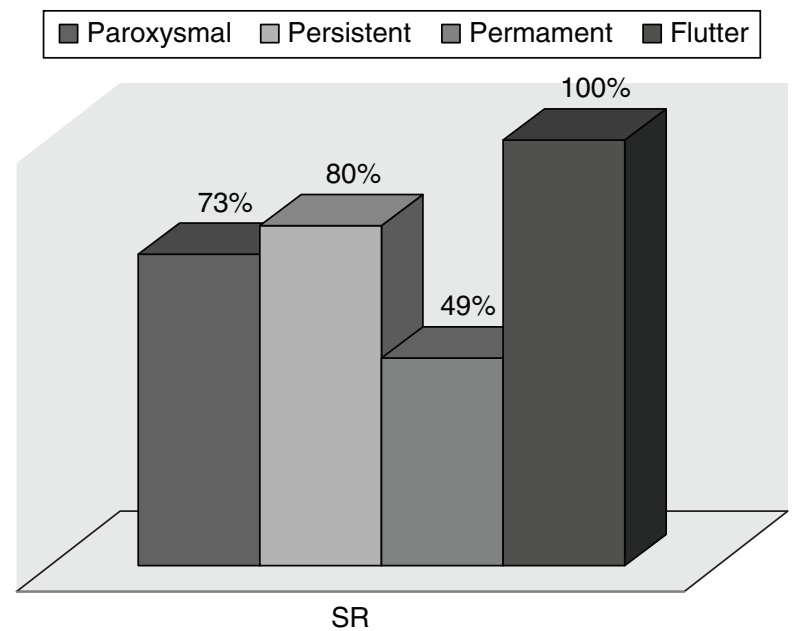

Fig. 1 Freedom from atrial fibrillation for the entire population at discharge. SR: sinus rhythm

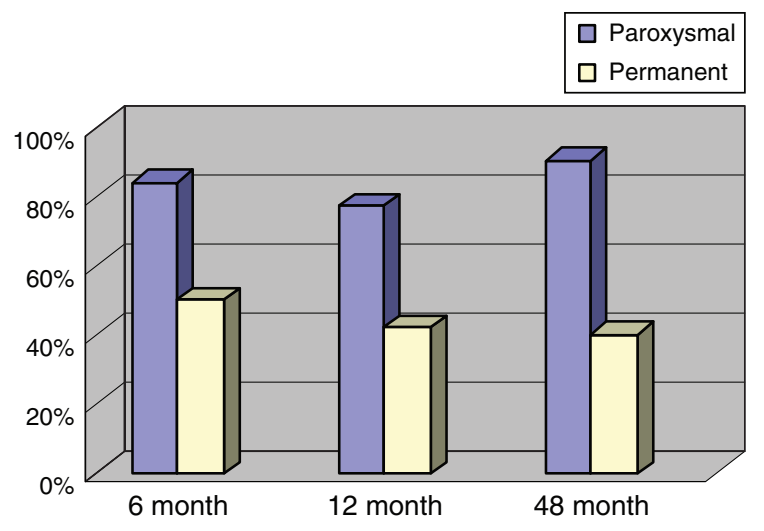

Fig. 2 Long-term surveillance of freedom of atrial fibrillation after high intensity focused ultrasound ablation: 6 months, 12 months and 48 months after ablation.

therapy, $57 \%$ of patients with amiodarone and $55 \%$ of patients without antiarrhythmics were presented with SR at discharge, respectively $67 \%$ vs. $65 \%$ vs. $69 \%$ at 6 month visit. In patients with $\beta$-blocker therapy were noted improved outcome by 16 percentage points, in amiodarone by 8 percentage points and in patients without antiarrhythmics by 14 percentage points.

Better results were seen in patients undergoing isolated mitral valve surgery with $72 \%$ (13) and coronary artery bypass surgery (CABG), who presented with $64 \%$ (21) freedom from AF. Patients with aortic valve replacement were associated with a lower success rate, with $53 \%$ (18) freedom from AF.

LA-size and AF-duration were significant predictors for a successful HIFU ablation. In our population, 78 patients had an LA diameter of $<50 \mathrm{~mm} ; 67 \%$ of these

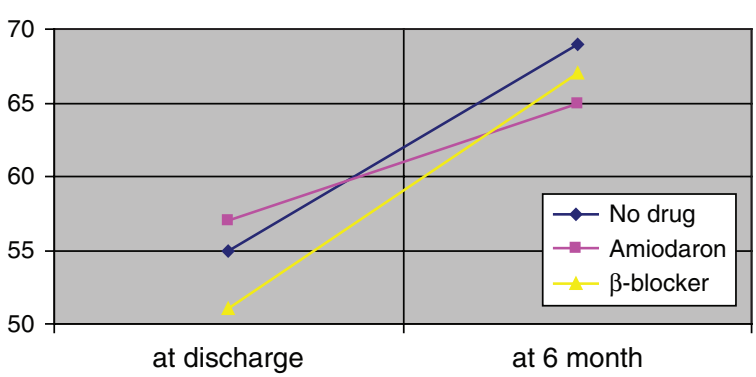

Fig. 3 Impact of antiarrhythmics, results at discharge, and 6 months after ablation.

patients were restored to SR, and $81 \%$ were in patients with paroxysmal AF (Fig. 4). Best results were achieved in patients with paroxysmal AF: AF-duration $<6$ months and LA $<50 \mathrm{~mm}$. Although it was a small group, all four patients were free of AF.

\section{Discussion}

$\mathrm{AF}$ has been identified as a risk factor for reduced long-term survival. ${ }^{3)}$ The 10-year survival of patients undergoing coronary bypass surgery without conversion of AF into SR is reduced by $24 \%$. In this study, the median survival of patients with AF was 8.7 years versus 14 years for those without it. ${ }^{14)}$

Our conversion rate was lower $(62 \%)$ than in previously published reports $(85 \%){ }^{15-18)}$ Our study population was not strictly selected towards predictors for successful ablation. In reality, every consecutive patient who wanted to participate was included, which may be related to the higher proportion of permanent $\mathrm{AF}$ and lower of paroxysmal and persistent AF. The better results of HIFU ablation in concomitant with Mitral Valve Surgery are supposedly to explain by the additional incision of the LA (atriotomy).

Reasons for the method's failure are still unknown. The device consists of a circular ring, so there is no lack of power on any place, but due to different thickness of the wall of the atrium there might be some areas of missing transmurality of coagulation. Imaginable answers of these problems are f.e. a repetition of the ablation algorithm after device rotating or a secondary trans-catheter ablation of remaining gaps in the ablation line. Further studies have to be done concerning this matter.

We found that antiarrhythmics after ablation could increase the success in restoration of SR. Though it was not significant, nevertheless, we recommend antiarrhythmics for the maintenance of SR. In the 2-year follow-up, 


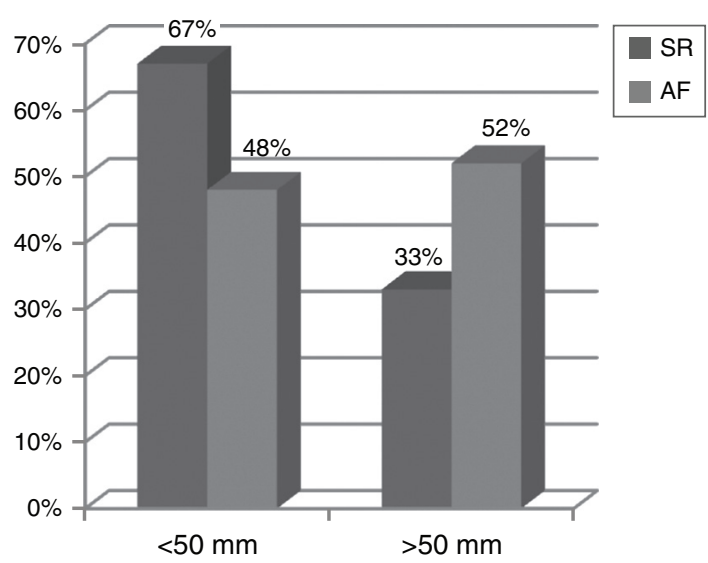

Fig. 4 Influence of LA size on results of ablation. SR: sinus rhythm; AF: atrial fibrillation; LA: left atrium.

we observed symptomatic AF recurrence in two patients by withdrawal of beta blocker. In both cases, hospitalisation was necessary.

After intravenous application of a $\beta$-blocker, both patients were converted spontaneously to SR. To maintain a stable SR, we recommend, if the patient can tolerate it, at least a small dosage of AAD. These would treat even the hypertension, which is a causative agent of AF. ${ }^{19)}$ Roux, et al. found that AAD treatment during the first 6 weeks after AF ablation is well tolerated and reduces the incidence of clinically significant atrial arrhythmias and need for cardioversion/hospitalization for arrhythmia management. ${ }^{20)}$

Despite the better results reported with small atrial size, shorter AF duration, recent joint guidelines on surgical ablation have recommended that all patients undergoing other cardiac surgery should be considered for $\mathrm{AF}$ ablation, if the risk of adding the procedure is low and a reasonable chance for success is expected. ${ }^{7)}$ Meta-analysis from Cheng, et al. including 4647 patients showed that, in patients with persistent or permanent AF who present for cardiac surgery, the addition of surgical AF ablation led to a significantly higher rate of SR $(\sim 70 \%)$ compared with cardiac surgery alone $(\sim 20 \%)$ at 1 year follow-up. ${ }^{18)}$

Excellent safety of Epicor HIFU-based System has been amply justified by recent publications. ${ }^{15-17)}$ It does not increase the operative duration, and during HIFU ablation the surgeon will perform the procedure for the commencement of cardiopulmonary bypass.

All patients with AF undergoing cardiac surgery should be considered for ablation and the technique should be made available at cardiac surgery centres. Patients with long term duration of $\mathrm{AF}$ and pronounced enlarged LA can be excluded.

\section{Limitations}

For rhythm evaluation in our study, only 12-lead ECG, clinical examination and standard 24-hour ECG registries were used, but no implantable ECG loop recorders, which have been shown to improve the completeness of follow-up data in other studies. ${ }^{21)}$ Follow-up did not include echocardiographic investigation of LA function, which also limits the completeness of the data. Our data represent only a single centre experience.

\section{Conclusion}

AF-Treatment with HIFU Ablation during concomitant cardiac surgery is a safe and effective procedure restoring SR especially in patients with either paroxysmal or persistent AF as well as in patients with smaller left atrial diameter. No significant difference was noted between antiarrhythmic and non drug groups regarding restoration and maintainance of SR, however, better results were achieved by the $\beta$-blocker.

\section{Disclosure Statement}

All authors have no conflict of interest.

\section{References}

1) Sack S. Epidemiology of atrial fibrillation. Herz 2002; 27: 294-300. (in German)

2) European Heart Rhythm Association; European Association for Cardio-Thoracic Surgery, Camm AJ, et al. Guidelines for the management of atrial fibrillation: the Task Force for the Management of Atrial Fibrillation of the European Society of Cardiology (ESC). Eur Heart J 2010; 31: 2369-429.

3) Calkins H, Brugada J, Packer DL, et al. HRS/EHRA/ ECAS expert consensus statement on catheter and surgical ablation of atrial fibrillation: recommendations for personnel, policy, procedures and follow-up. A report of the Heart Rhythm Society (HRS) Task Force on Catheter and Surgical Ablation of Atrial Fibrillation developed in partnership with the European Heart Rhythm Association (EHRA) and the European Cardiac Arrhythmia Society (ECAS); in collaboration with the American College of Cardiology (ACC), American Heart Association (AHA), and the Society of Thoracic Surgeons (STS). Endorsed and approved 
by the governing bodies of the American College of Cardiology, the American Heart Association, the European Cardiac Arrhythmia Society, the European Heart Rhythm Association, the Society of Thoracic Surgeons, and the Heart Rhythm Society. Europace 2007; 9: 335-79.

4) Wolf PA, Abbott RD, Kannel WB. Atrial fibrillation as an independent risk factor for stroke: the Framingham Study. Stroke 1991; 22: 983-8.

5) Le Heuzey JY, Paziaud O, Piot O, et al. Cost of care distribution in atrial fibrillation patients: the COCAF study. Am Heart J 2004; 147: 121-6.

6) Ringborg A, Nieuwlaat R, Lindgren P, et al. Costs of atrial fibrillation in five European countries: results from the Euro Heart Survey on atrial fibrillation. Europace 2008; 10: 403-11. Epub 2008 Mar 7.

7) Kim MH, Johnston SS, Chu BC, et al. Estimation of total incremental health care costs in patients with atrial fibrillation in the United States. Circ Cardiovasc Qual Outcomes 2011; 4: 313-20. Epub 2011 May.

8) Cox JL. The surgical treatment of atrial fibrillation. IV. Surgical technique. J Thorac Cardiovasc Surg 1991; 101: 584-92.

9) Jaïs P, Haïssaguerre M, Shah DC, et al. A focal source of atrial fibrillation treated by discrete radiofrequency ablation. Circulation 1997; 95: 572-6.

10) Benussi S, Nascimbene S, Agricola E, et al. Surgical ablation of atrial fibrillation using the epicardial radiofrequency approach: mid-term results and risk analysis. Ann Thorac Surg 2002; 74: 1050-6; discussion 1057.

11) Knaut M, Tugtekin SM, Jung F, et al. Microwave ablation for the surgical treatment of permanent atrial fibrillation-a single centre experience. Eur J Cardiothorac Surg 2004; 26: 742-6.

12) Williams MR, Casher JM, Russo MJ, et al. Laser energy source in surgical atrial fibrillation ablation: preclinical experience. Ann Thorac Surg 2006: 82: 2260-4.

13) Milla F, Skubas N, Briggs WM, et al. Epicardial beating heart cryoablation using a novel argon-based cryoclamp and linear probe. J Thorac Cardiovasc Surg 2006; 131: 403-11.

14) Quader MA, McCarthy PM, Gillinov AM, et al. Does preoperative atrial fibrillation reduce survival after coronary artery bypass grafting? Ann Thorac Surg 2004; 77: 1514-22; discussion 1522-4.

15) Ninet J, Roques X, Seitelberger R, et al. Surgical ablation of atrial fibrillation with off-pump, epicardial, high-intensity focused ultrasound: results of a multicenter trial. J Thorac Cardiovasc Surg 2005; 130: 803-9.

16) Groh MA, Binns OA, Burton HG 3rd, et al. Ultrasonic cardiac ablation for atrial fibrillation during concomitant cardiac surgery: long-term clinical outcomes. Ann Thorac Surg 2007; 84: 1978-83.

17) Schopka S, Schmid C, Keyser A, et al. Ablation of atrial fibrillation with the Epicor system: a prospective observational trial to evaluate safety and efficacy and predictors of success. J Cardiothorac Surg 2010; 5: 34.

18) Cheng DC, Ad N, Martin J, et al. Surgical ablation for atrial fibrillation in cardiac surgery: a meta-analysis and systemic review. Innovations (Phila) 2010; 5: 84-96.

19) Healey JS, Connolly SJ. Atrial fibrillation: hypertension as a causative agent, risk factor for complications, and potential therapeutic target. Am J Cardiol 2003; 91: 9G-14G.

20) Roux JF, Zado E, Callans DJ, et al. Antiarrhythmics after ablation of atrial fibrillation (5A Study). Circulation 2009; 120: 1036-40.

21) Task Force members, Brignole M, Vardas P, et al. Indications for the use of diagnostic implantable and external ECG loop recorders. Europace 2009; 11: 671-87. 